\title{
Biomassa e atividade microbiana do solo em sistemas de produção olerícola orgânica e convencional
}

\author{
Biomass and soil microbial activity on horticulture organic and conventional crop systems
}

\author{
Rogério Otávio Schmidt ${ }^{\mathrm{I}}$ Ronei Sandri Sana ${ }^{\mathrm{I}}$ Felipe Krüger Leal ${ }^{\mathrm{I}}$ Robson Andreazza ${ }^{\mathrm{II}}$ \\ Flávio Anastácio de Oliveira Camargo ${ }^{\mathrm{I}}$ Egon José Meurer ${ }^{\mathrm{I}}$
}

\section{RESUMO}

\begin{abstract}
O cultivo de olerícolas pode ser produzido no sistema convencional utilizando defensivos agrícolas $e$ fertilizantes sintéticos, ou no sistema orgânico, quando não há a utilização de defensivos sintéticos e tem como principal fonte de adubação, resíduos orgânicos. Este trabalho teve como objetivo avaliar a biomassa e a atividade microbiana do solo em sistemas de cultivo orgânico e convencional com suas respectivas áreas de referência. Para isso, foram coletadas amostras de solo em três propriedades agrícolas com sistema de cultivo convencional e outras três com sistema de cultivo orgânico, além de suas respectivas áreas de referência. Foram determinados o carbono e nitrogênio microbiano e total, e a respiração basal após 57 dias de incubação. A maior variação na taxa de respiração basal entre a área de produção e a área de referência foi observada no sistema orgânico. Os teores de nutrientes no solo, carbono e nitrogênio microbianos foram maiores nas áreas de produção do que os encontrados nas áreas de referência.
\end{abstract}

Palavras-chave: liberação de $\mathrm{CO}_{2}$, atividade microbiana, nitrogênio e carbono microbiano.

\section{ABSTRACT}

The horticultural crops can be produced with the conventional system using pesticides and synthetic fertilizers, or organic system where there is no use of synthetic pesticides and its main source of nutrients are organic wastes. This study aimed to assess the biomass and soil microbial activity in organic production systems and conventional with their respective reference areas. In this study, soil samples were sampled in three farms with conventional tillage and three other with organ system, and their respective reference areas. It was determined total carbon and nitrogen and microbial, and microbial activity after
57 days of incubation. The greatest variation in basal respiration rate between the respective production area and reference area was observed in the organic system. The nutrients in the soil microbial carbon and nitrogen were higher in the production areas than those in the reference areas.

Key words: $\mathrm{CO}_{2}$ evolution, microbial activity, microbial carbon and nitrogen.

\section{INTRODUÇÃO}

A produção olerícola convencional gera grande impacto sobre o ambiente, devido ao intenso revolvimento do solo, aplicação de fertilizantes e agrotóxicos ao solo. Os sistemas mais utilizados na produção olerícola são os sistemas de produção convencional e orgânica. Esses sistemas se diferem em vários aspectos de produção, como no controle de pragas, em que o sistema de produção orgânica não utiliza defensivos sintéticos, enquanto que o sistema convencional utiliza intensivamente agroquímicos no controle de pragas e plantas daninhas (MELLO et al., 2003). No entanto, o sistema orgânico utiliza como principal fonte de adubação grandes quantidades de fertilizante orgânico, principalmente cama de aves, cuja recomendação é elaborada pela Comissão de Química e Fertilidade do solo do Estado do Rio Grande do Sul e Santa Catarina, a qual recomenda aplicar em média $12 \mathrm{t}$ $\mathrm{ha}^{-1}$ ano $^{-1}$, dependendo das condições de solo e da fertilidade (COMISSÃO..., 2004).

\footnotetext{
'Universidade Federal do Rio Grande do Sul (UFRGS), Porto Alegre, RS, Brasil. correspondência.

"Universidade Federal de Pelotas (UFPel), 96010-280, Pelotas, RS, Brasil. E-mail: robsonandreazza@yahoo.com.br. Autor para 
Para verificar a relação entre o sistema de produção e o meio ambiente, podem ser adotados diversos critérios. Dentre eles, os indicadores considerados como mais sensíveis à mudança ambiental são os indicadores biológicos (ARAÚJO \& MONTEIRO, 2007), dos quais, os mais indicados para monitorar possíveis mudanças no solo são: a biomassa e a atividade microbiana (ARAÚJO \& MONTEIRO, 2007; GLAESER et al., 2010; SPARLING, 1997). Segundo ARAÚJO \& MONTEIRO (2007), o monitoramento da matéria orgânica do solo (MOS) constitui-se de um indicador importante da qualidade do sistema, tendo a mesma relação com a fertilidade, estrutura e estabilidade do solo.

A biomassa microbiana é responsável pela decomposição e mineralização de resíduos vegetais e orgânicos, e utiliza esses materiais como fonte de energia e nutrientes para sua manutenção e multiplicação (GAMA-RODRIGUES \& GAMA-RODRIGUES, 2008). A sua quantificação permite avaliar alterações na quantidade de matéria orgânica causadas pelo sistema de cultivo (MERCANTE et al., 2008), o que permite determinar possíveis mudanças na ciclagem de nutrientes e produtividade do sistema (TÓTOLA \& CHAER, 2002). Assim, este trabalho teve como objetivo avaliar o efeito de dois sistemas de produção olerícola sobre os teores de carbono e nitrogênio orgânico e total do solo, carbono e nitrogênio microbiano e a atividade microbiana do solo das diferentes áreas de produção.

\section{MATERIAL E MÉTODOS}

O efeito dos diferentes sistemas de produção olerícola sobre a microbiota do solo foi avaliado. Seis propriedades de produção olerícola foram utilizadas, sendo três de produção convencional (PC) e três de produção orgânica (PO), além de suas respectivas áreas de referência $(\mathrm{R})$. Essas áreas de referência estão localizadas próximas às áreas de produção e não são manejadas com a mesma intensidade das áreas de produção, sendo cobertas por vegetação nativa, pastagem cultivada ou pomar abandonado, permitindo assim comparar o efeito do sistema produtivo em cada local. As seguintes áreas de referência estão compostas por: propriedades PO1 e PC3, que são áreas de campo nativo; PC1 e PC2 são de mata nativa; e PO2 e PO3 têm como vegetação um pomar abandonado e pastagem cultivada, respectivamente. O solo das propriedades PO1 e PC2 foram classificados como PLANOSSOLO Háplico Distrófico gleissólico; e o solo das propriedades PO2, PO3, PC1 e PC3 foram classificados como ARGISSOLO Vermelho Distrófico arênico (EMBRAPA, 1999). A relação textural dos solos das áreas de produção e referência das propriedades $\mathrm{PO} 1$, PO2, PC1, PC2 e PC3 é franco arenosa, e da área PO3 é areia franca. O presente estudo desenvolveu-se na zona rural do município de Novo Hamburgo/RS. Em cada propriedade, no mês de novembro/2011, foram coletadas 20 subamostras de cada solo com trado de rosca na profundidade de $0-20 \mathrm{~cm}$, distribuídas conforme metodologia descrita pela EMBRAPA (2009) para amostragem de solos em áreas de produção olerícola. Após a coleta e homogeneização dos solos, foram retiradas algumas subamostras para realizar as análises químicas. Estas foram secas ao ar e tamisadas em peneira de malha de $2 \mathrm{~mm}$, para então determinar, conforme metodologia descrita por TEDESCO et al. (1995), os seguintes atributos: índice SMP; fósforo, potássio e sódio disponíveis; cálcio, magnésio, alumínio e manganês trocáveis; zinco e cobre extraíveis. Os teores de areia, silte e argila foram determinados pelo método da pipeta, conforme descrito pela EMBRAPA (1997). Os resultados da análise ficoquímica dos solos estão descritos na tabela 1.

Para avaliação da atividade microbiana, as subamostras coletadas na profundidade de $0-20 \mathrm{~cm}$ foram acondicionadas em sacos de PVC de 5L, homogeneizadas, formando uma amostra composta. Após cinco amostras de $100 \mathrm{~g}$ de solo fresco em condição natural foram pesados e acondicionadas em frascos de vidro hermeticamente fechados de volume de 1L, para avaliar a atividade microbiana dos solos de diferentes áreas de produção e suas respectivas áreas de referência. A respiração basal do solo foi determinada pela quantificação do dióxido de carbono $\left(\mathrm{CO}_{2}\right)$ total liberado no processo de respiração microbiana, a partir de $100 \mathrm{~g}$ de solo acondicionadas nos vidros, durante 57 dias de incubação à temperatura ambiente (temperatura média de $28^{\circ} \mathrm{C} \pm 5$ ). Cada amostra foi incubada nos frascos de $1 \mathrm{~L}$, providos de recipientes contendo $10 \mathrm{~mL}$ de $0,5 \mathrm{M} \mathrm{NaOH}$ para captação do $\mathrm{CO}_{2}$ liberado. Após a incubação, os recipientes com $\mathrm{NaOH}$ foram retirados e receberam $1 \mathrm{~mL}$ de $\mathrm{BaCl}_{2}(30 \%)$ e 3 gotas de fenolftaleína para titulação com $0,25 \mathrm{M} \mathrm{HCl}$ padronizado. As determinações foram realizadas aos 3 , $9,15,22,29,36,43,50$ e 57 dias após o início da incubação. A liberação do $\mathrm{CO}_{2}$ foi calculada de acordo comSTOTZKY (1965).

O carbono da biomassa microbiana foi determinado através da metodologia citada por VANCE et al. (1987), utilizando-se em substituição ao método de fumigação-extração. Então, utilizou-se o método irradiação-extração, com forno de microondas marca Brastemp maxi, modelo BMS35BBHNA, tensão de alimentação $120 \mathrm{~V}(60 \mathrm{~Hz})$, frequência de $2.450 \mathrm{MHz}$, por 
Tabela 1 - Caracterização físico-química da camada superficial $(0-20 \mathrm{~cm})$ dos solos coletados das propriedades de produção olerícola orgânica e convencional.

\begin{tabular}{|c|c|c|c|c|c|c|c|c|c|c|}
\hline Produtor & Área & $\begin{array}{l}\mathrm{TC} \\
\text { Anos }\end{array}$ & $\begin{array}{l}\text { Ad. Org. } \\
\text { - }\end{array}$ & $\begin{array}{l}\text { Ad. Quím. } \\
\text { nno-1 }\end{array}$ & $\mathrm{pH} \mathrm{H} \mathrm{H}_{2} \mathrm{O}$ & SMP & $\begin{array}{c}\text { M.O. } \\
\%\end{array}$ & Areia $a_{\text {total }}$ & $\begin{array}{l}\text { Silte } \\
\mathrm{g} \mathrm{kg}^{-1}\end{array}$ & Argila \\
\hline \multirow{2}{*}{ PO1 } & Produção & 22 & 10.000 & - & 6,6 & 6,8 & 2,9 & 735 & 205 & 61 \\
\hline & Referência & - & - & - & 5,1 & 5,9 & 1,4 & 733 & 192 & 75 \\
\hline \multirow{2}{*}{$\mathrm{PO} 2$} & Produção & 4 & 36.000 & - & 6,0 & 6,8 & 2,8 & 777 & 168 & 56 \\
\hline & Referência & - & - & - & 5,2 & 6,1 & 1,1 & 740 & 177 & 83 \\
\hline \multirow{2}{*}{ PO3 } & Produção & 20 & 13.000 & - & 6,3 & 7,1 & 1,1 & 827 & 134 & 38 \\
\hline & Referência & - & - & - & 6,4 & 6,9 & 0,8 & 836 & 124 & 40 \\
\hline \multirow{2}{*}{ PC1 } & Produção & 11 & 16.000 & 100 & 5,8 & 6,6 & 1,6 & 716 & 182 & 101 \\
\hline & Referência & - & - & - & 5,3 & 5,7 & 2,1 & 669 & 181 & 150 \\
\hline \multirow{2}{*}{ PC2 } & Produção & 20 & 20.000 & 220 & 5,4 & 6,4 & 1,6 & 751 & 147 & 102 \\
\hline & Referência & - & - & - & 4,6 & 5,6 & 2,1 & 777 & 137 & 87 \\
\hline \multirow{2}{*}{ PC3 } & Produção & 21 & 21.000 & 150 & 6,3 & 6,8 & 1,9 & 760 & 121 & 119 \\
\hline & Referência & - & - & - & 5,3 & 5,7 & 1,8 & 665 & 114 & 221 \\
\hline Produtor & Área & $\mathrm{Al}^{3+}{ }_{\text {troc. }}$ & $\mathrm{Ca}_{\text {troc. }}$ & $\mathrm{Mg}_{\text {troc. }}$ & $\mathrm{Al}+\mathrm{H}$ & $\mathrm{Na}$ & K & $\mathrm{P}$ & $\mathrm{Cu}$ & $\mathrm{Zn}$ \\
\hline \multirow{2}{*}{ PO1 } & Producão & 0,0 & 9.6 & 2.7 & 1.8 & 0,12 & 100 & 639 & 2,2 & 562 \\
\hline & Referência & 0,5 & 0,6 & 0,3 & 4,9 & 0,02 & 0,11 & 3,8 & 1,4 & 1,1 \\
\hline \multirow{2}{*}{$\mathrm{PO} 2$} & Produção & 0,0 & 4,8 & 1,5 & 1,8 & 0,06 & 0,40 & 129 & 2,9 & 12,7 \\
\hline & Referência & 0,1 & 1,4 & 0,6 & 4,1 & 0,02 & 0,13 & 32 & 1,5 & 2,6 \\
\hline \multirow{2}{*}{ PO3 } & Produção & 0,0 & 2,8 & 1,0 & 1,4 & 0,06 & 0,63 & 167 & 2,2 & 15,6 \\
\hline & Referência & 0,0 & 1,9 & 0,7 & 1,6 & 0,02 & 0,13 & 87 & 1,7 & 5,1 \\
\hline \multirow{2}{*}{ PC1 } & Produção & 0,0 & 5,8 & 1,0 & 2,2 & 0,10 & 1,11 & 424 & 3,0 & 31,1 \\
\hline & Referência & 0,1 & 1,7 & 2,0 & 6,2 & 0,09 & 0,48 & 12 & 1,8 & 3,8 \\
\hline \multirow{2}{*}{ PC2 } & Produção & 0,0 & 4,6 & 1,1 & 2,8 & 0,12 & 0,89 & 256 & 4,6 & 11,8 \\
\hline & Referência & 0,5 & 0,8 & 0,5 & 6,9 & 0,04 & 0,14 & 10 & 1,3 & 1,3 \\
\hline \multirow[b]{2}{*}{ PC3 } & Produção & 0,0 & 7,0 & 1,8 & 1,7 & 0,18 & 0,64 & 734 & 1,4 & 53,3 \\
\hline & Referência & 0,4 & 1,9 & 0,7 & 6,2 & 0,03 & 0,15 & 5 & 2,1 & 1,4 \\
\hline
\end{tabular}

TC = tempo de cultivo no sistema de produção utilizado atualmente; Ad. Org. = adubação orgânica aplicada nas áreas de produção olerícola orgânica e convencional ao longo do ano; Ad. Quím. = quantidade de fertilizante mineral (5-20-20) aplicado nas áreas de produção olerícola convencional; Areia, Silte e Argila dispersos por solução de NaOH de $1 \mathrm{M}$ e determinados pelo método da Pipeta (EMBRAPA, 1997); Al, Ca e Mg extraídos com KCl 1M; Na, K e P determinados por Mehlich 1; Cu e Zn extraídos por HCl 0,1M.

três minutos, totalizando $2,52 \times 10^{5} \mathrm{~J}$ de energia emitida durante o tempo de irradiação, atingindo os microrganismos e provocando a liberação dos compostos celulares, conforme sugerido por FERREIRA et al. (1999). O carbono e o nitrogênio da biomassa microbiana (Cmic e Nmic) foram extraídos por uma solução de $0,5 \mathrm{M} \mathrm{K}_{2} \mathrm{SO}_{4}$. O carbono foi determinado por combustão via úmida e o nitrogênio pelo método de Kjedhal (TEDESCO et al., 1995).

Os valores do carbono da biomassa microbiana do solo (BMS-C) foram calculados segundo metodologia descrita por SPARLING \& WEST (1988) e 
os valores de nitrogênio da biomassa microbiana do solo (BMS-N) foram calculados segundo metodologia descrita por BROOKES et al. (1982). Para determinação do teor de carbono orgânico do solo, utilizou-se o método de combustão úmida WALKLEY-BLACK, modificado por TEDESCO et al. (1995), e o teor de Nitrogênio total no solo foi determinado segundo metodologia descrita por TEDESCO et al. (1995).

A análise dos dados foi feita pela análise da variância individual e conjunta dos dados, o teste de significância foi realizado pelo teste ANOVA, ao nível de 5\% de probabilidade. Para os dados de evolução de $\mathrm{CO}_{2}$, utilizou-se o modelo de análise de variância (anova) para medidas repetidas, por meio do programa estatístico SAS, uma vez que os dados foram repetidamente obtidos nos mesmos vasos em dias subsequentes.

\section{RESULTADOS E DISCUSSÃO}

A atividade microbiana do solo dos diferentes sistemas de cultivos durante 57 dias de incubação são apresentadas na figura 1. Em geral, os solos das áreas de produção apresentaram maior taxa de liberação de $\mathrm{CO}_{2}$, comparados aos solos das áreas de referência ao longo do tempo (Figura 1), tendo apenas a área de produção $\mathrm{PC} 1$ apresentado valor inferior à área de referência, a qual diferiu a partir de 29 dias, não diferindo $(\mathrm{P}>0,05)$ na fase inicial do experimento. Nos solos das áreas de produção orgânica, como PO1 e PO2, foi observado o maior incremento na atividade microbiana, quando comparado as suas respectivas áreas de referência, apresentando valores totais de 70,1 e 56,9mg $\mathrm{CO}_{2} 100 \mathrm{~g} \mathrm{solo}^{-1}$, respectivamente. Já nos solos de

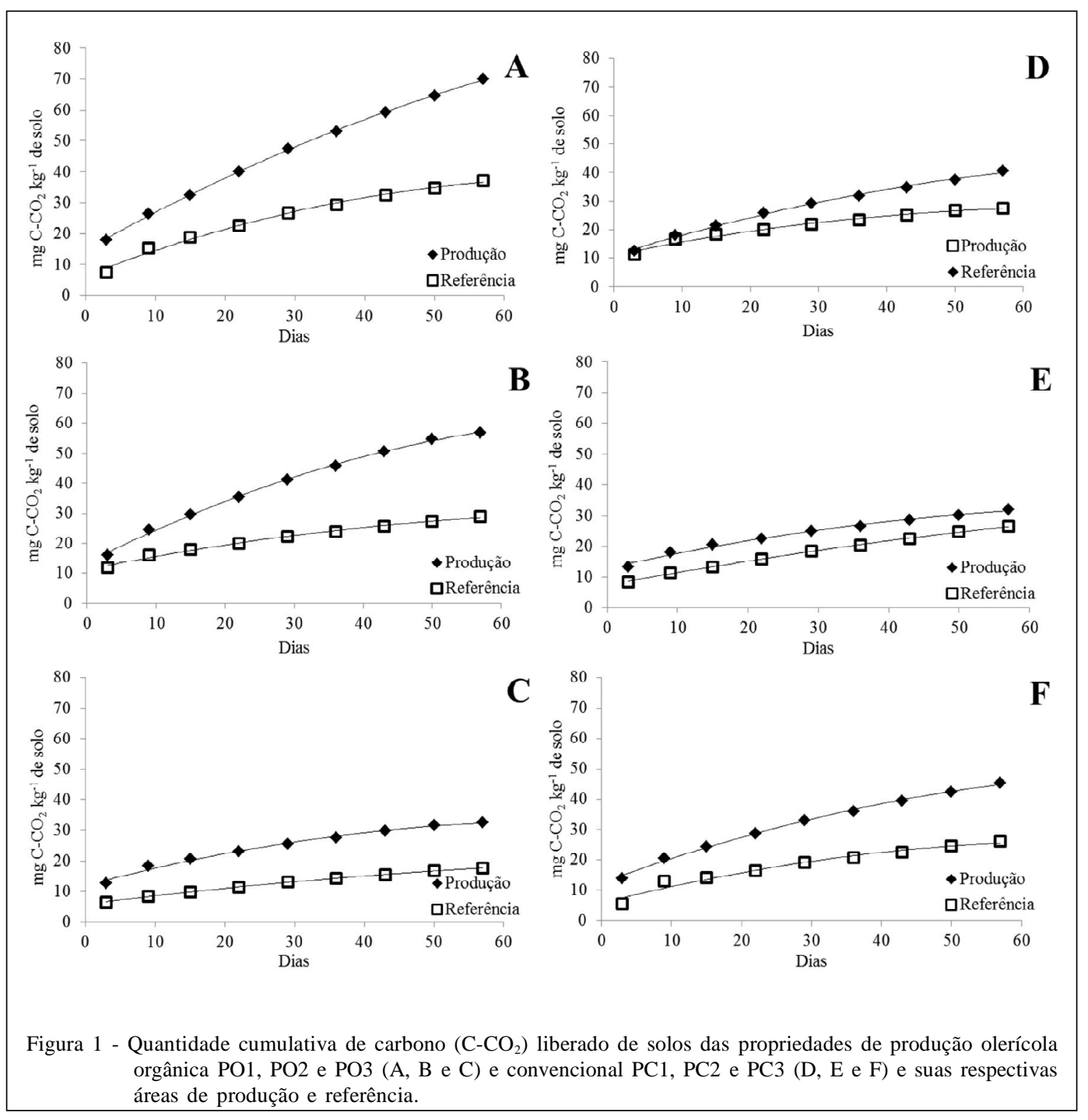

Ciência Rural, v.43, n.2, fev, 2013. 
produção convencional $\mathrm{PC} 1$ e $\mathrm{PC} 2$, foi observado um menor variação na atividade microbiana do solo em relação as suas áreas de referência, apresentado valores de 27,4 e $31,9 \mathrm{mg} \mathrm{CO}_{2} 100 \mathrm{~g} \mathrm{solo}^{-1}$, respectivamente. Segundo GAMA-RODRIGUES \& GAMA-RODRIGUES (2008), de modo geral, quanto menor a taxa de respiração, mais eficiente será a microbiota na incorporação de carbono à biomassa. No entanto, MELERO et al. (2006) afirma que a adição de resíduos orgânicos, como a cama de aves, promove maior atividade microbiana do que a adubação química, possibilitando assim maior disponibilidade de nutrientes em sistema de manejo orgânico do que o convencional. Contudo, a inter-relação entre diferentes tipos de manejo, tipos de solo e características próprias dos solos pode favorecer ou reduzir a atividade microbiana do solo, dependendo da condição ambiental e da inter-relação de fatores em um determinado ambiente.

Essas diferenças de atividade microbiana entre as áreas de produção e as áreas de referência podem ter ocorrido devido à baixa fertilidade natural das áreas de referência e menor fonte de energia, principalmente nitrogênio e matéria orgânica, que é a principal fonte de energia (Tabelas 1 e 2). Um fator que promoveu o aumento da liberação de $\mathrm{C}-\mathrm{CO}_{2}$ nas áreas de produção orgânica é a adição de adubação orgânica, a qual, segundo SILVA et al. (2010), em trabalho comparando a aplicação de diferentes adubos orgânicos sobre a emissão de $\mathrm{CO}_{2}$, a cama de aves foi a que resultou em uma maior emissão de $\mathrm{CO}_{2}$, sendo este fertilizante orgânico o mais utilizado nas áreas de produção olerícola.

O uso de herbicidas no controle de daninhas pode provocar a diminuição na atividade microbiana. Isso é confirmado por TIRONI et al. (2009) em trabalho avaliando o efeito de herbicidas na atividade microbiana do solo. Os autores observaram que houve efeito negativo do uso de herbicida no controle de plantas daninhas sobre a microbiota do solo. Esse também pode ser um dos fatores que influenciou na menor diferença nos resultados de liberação de $\mathrm{C}-\mathrm{CO}_{2}$ nas áreas de produção convencional, com relação às áreas de produção orgânica.

Em relação ao nitrogênio total (NT) e ao carbono orgânico total (COT), as áreas de produção orgânica diferiram estatisticamente $(\mathrm{P} \leq 0,05)$ das áreas de referência, tendo sido superior em todas as áreas (Tabela 2). No geral, os valores de NT não diferiram

Tabela 2 - Respiração Basal (RB), nitrogênio total (NT), carbono orgânico total (COT), nitrogênio da biomassa microbiana (BMS-N) e carbono da biomassa microbiana do solo (BMS-C) em três solos de sistema de produção orgânica (PO) e em três solos de sistema de produção convencional (PC).

\begin{tabular}{|c|c|c|c|c|c|c|}
\hline Sistema & Área & $\mathrm{RB}$ & NT & COT & BMS-N & BMS-C \\
\hline \multirow{3}{*}{ PO1 } & & $\mathrm{mg} 100 \mathrm{~g}^{-1}$ solo & \multicolumn{2}{|c|}{ - } & \multicolumn{2}{|c|}{ - $\mathrm{mg} \mathrm{kg}^{-1}$ solo } \\
\hline & Produção & $70,1 \mathrm{a}^{*}$ & $1,43 \mathrm{a}^{* *}$ & $16,70 \mathrm{a}^{* *}$ & $53,68 \mathrm{a}^{* *}$ & $212,15 \mathrm{~ns}^{* *}$ \\
\hline & Referência & $37,2 \mathrm{~b}$ & $0,47 \mathrm{~b}$ & $8,01 \mathrm{~b}$ & $25,38 \mathrm{~b}$ & $109,95 \mathrm{~ns}$ \\
\hline \multirow{2}{*}{$\mathrm{PO} 2$} & Produção & $56,9 \mathrm{a}$ & $1,20 \mathrm{a}$ & $16,07 \mathrm{a}$ & $3,12 \mathrm{~b}$ & $210,46 \mathrm{a}$ \\
\hline & Referência & $28,9 \mathrm{~b}$ & $0,46 \mathrm{~b}$ & $6,57 \mathrm{~b}$ & $15,13 \mathrm{a}$ & $103,71 \mathrm{~b}$ \\
\hline \multirow{2}{*}{$\mathrm{PO} 3$} & Produção & $32,5 \mathrm{a}$ & $0,54 \mathrm{a}$ & $6,43 \mathrm{a}$ & $11,52 \mathrm{~ns}$ & 94,64 a \\
\hline & Referência & $17,7 \mathrm{~b}$ & $0,25 \mathrm{~b}$ & $4,39 \mathrm{~b}$ & $6,60 \mathrm{~ns}$ & $14,27 \mathrm{~b}$ \\
\hline \multirow{2}{*}{$\mathrm{PC} 1$} & Produção & $27,4 \mathrm{~b}$ & $0,74 \mathrm{~ns}$ & $9,02 \mathrm{~b}$ & $20,30 \mathrm{~b}$ & $142,27 \mathrm{~b}$ \\
\hline & Referência & $40,5 \mathrm{a}$ & $0,92 \mathrm{~ns}$ & $11,92 \mathrm{a}$ & $41,26 \mathrm{a}$ & $321,28 \mathrm{a}$ \\
\hline \multirow{2}{*}{$\mathrm{PC} 2$} & Produção & $31,9 \mathrm{a}$ & $0,66 \mathrm{~ns}$ & $9,31 \mathrm{~b}$ & $16,53 \mathrm{~ns}$ & $124,31 \mathrm{~ns}$ \\
\hline & Referência & $26,5 \mathrm{~b}$ & $0,77 \mathrm{~ns}$ & $11,98 \mathrm{a}$ & $26,51 \mathrm{~ns}$ & $188,35 \mathrm{~ns}$ \\
\hline \multirow{2}{*}{ PC3 } & Produção & $45,3 \mathrm{a}$ & $0,86 \mathrm{a}$ & $10,84 \mathrm{~ns}$ & $38,35 \mathrm{~ns}$ & $141,96 \mathrm{~ns}$ \\
\hline & Referência & $27,3 \mathrm{~b}$ & $0,65 \mathrm{~b}$ & $10,65 \mathrm{~ns}$ & $32,88 \mathrm{~ns}$ & $193,19 \mathrm{~ns}$ \\
\hline
\end{tabular}

*Análise de variância (ANOVA) para medidas repetidas, a 5\% de probabilidade de erro dentro da mesma propriedade, por meio do programa estatístico SAS. Letras iguais não diferem estatisticamente. ** Teste ANOVA a 5\% de probabilidade de erro dentro da mesma propriedade, comparando área de produção e referência. ns = não significativo. 
$(\mathrm{P}>0,05)$ nas áreas de referência das áreas de produção convencional, onde foi apresentado valor superior à área de referência apenas na área PC3. Em relação ao COT, as áreas PC1 e PC2 apresentaram valores inferiores $(\mathrm{P} \leq 0,05)$ às áreas de referência, onde apenas a área $\mathrm{PC} 3$ não diferiu $(\mathrm{P}>0,05)$ da área de referência.

O carbono da biomassa microbiana do solo (BMS-C) das áreas de produção orgânica, em sua maioria, foram superiores $(\mathrm{P} \leq 0,05)$ às áreas de referência, onde apenas a área PO1 não diferiu da área de referência. Em relação às áreas de produção convencional, as áreas $\mathrm{PC} 2$ e $\mathrm{PC} 3$ não diferiram $(\mathrm{P}>0,05)$ de suas respectivas áreas de referência, tendo a área $\mathrm{PC} 1$ apresentado valor de BMS-C menor $(\mathrm{P} \leq 0,05)$ do que sua área de referência. Em relação ao nitrogênio da biomassa microbiana do solo (BMS-N), a área PO1 foi superior $(\mathrm{P}=0,001845)$ à área de referência, tendo a área PO2 e PC1 apresentado valor inferior $(\mathrm{P} \leq 0,05)$, e as áreas PO3, PC2 e PC3 não diferiram $(\mathrm{P}>0,05)$ das suas respectivas áreas de referência (Tabela 2). Segundo GAMA-RODRIGUES \& GAMA-RODRIGUES (2008), a maior biomassa microbiana observada nas áreas de produção orgânica pode ser utilizada como um indicador de sustentabilidade do sistema de produção, quando este é comparado com a sua área de referência. SILVA et al. (2006) também observaram maior incremento nos teores de carbono e nitrogênio microbiano em áreas de cultivo orgânico, e redução destes em áreas de cultivo convencional, quando comparados às áreas de mata nativa.

Os resultados observados de aumento no conteúdo de COT, NT, RB, BMS-N e BMS-C em áreas de produção orgânica também têm sido observados por outros autores (MELERO et al., 2006; SAMPAIO et al., 2008), indicando que, nessas áreas, apresenta-se maior atividade da biomassa microbiana e manutenção do conteúdo de C orgânico.

\section{CONCLUSÃO}

No geral, as áreas de produção orgânica apresentaram maior atividade microbiana dos solos do que as áreas de produção convencional. $\mathrm{O}$ nitrogênio total e o carbono orgânico total foram superiores nas áreas de produção orgânica, enquanto que as áreas de produção convencional não diferiram estatisticamente e o nitrogênio total e o carbono orgânico total foi inferior nas áreas de produção, quando comparados às áreas de referência.

O nitrogênio da biomassa microbiana do solo das áreas de produção não apresentou diferenças significativas das áreas de referência. O carbono da biomassa microbiana do solo foi maior na maioria das áreas de produção orgânica, enquanto que as áreas de produção convencional não diferiram das áreas de referência. De maneira geral, o sistema de produção orgânica contribuiu para o aumento da biomassa microbiana do solo, já o sistema de produção convencional diminuiu a biomassa microbiana do solo, quando comparado às áreas de referência.

\section{AGRADECIMENTOS}

À Coordenação de Aperfeiçoamento Profissional de Nível Superior (CAPES), ao Conselho Nacional de Desenvolvimento Científico e Tecnológico (CNPq), pelo apoio financeiro e bolsas concedidas, e à EMATER/RS, pelo apoio e pela intermediação com os agricultores.

\section{REFERÊNCIAS}

ARAÚJO. A.S.F; MONTEIRO, R.T.R. Indicadores biológicos de qualidade do solo. Bioscience Journal, v.23. n.3, p.66-75, 2007. Disponível em: <http://www.seer.ufu.br/index.php/biosciencejournal/ article/view/6684/4403>. Acesso em: 18 jul. 2012.

BROOKES, P.C. et al. Measurement of microbial biomass phosphorus in soil. Soil Biology \& Biochemistry, v.14, n.4, p.319-329, 1982. Disponível em: <http://www.sciencedirect.com/ science/article/pii/0038071782900013>. Acesso em: 17 jul. 2012. doi: 10.1016/0038-0717(82)90001-3.

COMISSÃO DE QUÍMICA E FERTILIDADE DO SOLO - RS/ SC. Manual de adubação e calagem para os Estados do Rio Grande do Sul e de Santa Catarina. 10.ed. Porto Alegre: SBCS-CQFS, 2004. 400 p.

EMBRAPA. Centro Nacional de Pesquisa de Solos. Manual de métodos de análise de solos. 2. ed. rev. e atual. Rio de Janeiro, 1997. 212p.

EMBRAPA. Centro Nacional de Pesquisa de Solos. Sistema Brasileiro de Classificação de Solos. Brasília: Embrapa Produção de informação; Rio de Janeiro: Embrapa Solos, 1999. 412p.

EMBRAPA - Embrapa Solos. Manual de análises químicas de solos, plantas e fertilizantes. Brasília: Embrapa Informação Tecnológica; Brasília, DF: Embrapa Solos, 2009. 627p.

FERREIRA, A.S. et al. Utilização de microondas na avaliação da biomassa microbiana do solo. Revista Brasileira de Ciência do Solo, v.23, p.991-996, 1999. Disponível em: <http:// www.scielo.br/scielo.php?script $=$ sci_arttext \&pid $=S 0100$ 06832004000600006>. Acesso em: 16 jul. 2012. doi: http:// dx.doi.org/10.1590/S0100-06832004000600006.

GAMA-RODRIGUES, R.E.; GAMA-RODRIGUES, C.A. Biomassa microbiana e ciclagem de nutrientes. In:SANTOS, G.A. et al. Fundamentos da matéria orgânica do solo: ecossistemas tropicais e subtropicais. 2.ed. rev. e atual. Porto Alegre: Metrópole, 2008. p.159-182.

GLAESER, D.F. et al. Biomassa microbiana do solo sob sistemas de manejo orgânico em cultivos de café. Ensaios e Ciência: Ciências Biológicas, Agrárias e da Saúde, 2010. V.14. Disponível em: <http:/ /www.redalyc.org/src/inicio/ArtPdfRed.jsp?iCve=26019017009>. Acesso em: 16 jul. 2012. 
MELERO, S. et al. Chemical and biochemical properties in a silty loam soil under conventional and organic management. Soil Tillage Research, v.90, p.162-170, 2006. Disponível em: <http:/ /www.sciencedirect.com/science/article/pii/S0167198705002497>. Acessado em: 18 jul. 2012. doi: 10.1016/j.bbr.2011.03.031.

MELLO, J.C. et al. Efeito do cultivo orgânico e convencional sobre a vida-de-prateleira de alface americana (Lactuca sativa L.) minimamente processada. Ciência e Tecnologia dos Alimentos, v.23, n.3, p.418-426, 2003. Disponível em: $<$ http://www.scielo.br/scielo.php?pid=S0101-20612003 000300022\&script=sci_arttext $>$. Acesso em: 18 jul. 2012. doi: $10.1590 / \mathrm{S} 0101-20612003000300022$.

MERCANTE, F.M. et al. Biomassa microbiana, em um Argissolo Vermelho, em diferentes coberturas vegetais, em área cultivada com mandioca. Acta Scientiarum: Agronomy, v.30, n.4, p.479485, 2008. Disponível em: <http://periodicos.uem.br/ojs/ index.php/ActaSciAgron/article/view/5301/5301>. Acesso em: 14 fev. 2012. doi: 10.4025/actasciagron.v30i4.5301.

SAMPAIO, D.B. et al. dos. Avaliação de indicadores biológicos de qualidade do solo sob sistemas de cultivo convencional e orgânico de frutas. Ciência Agrotécnica, v.32, n.2, p.353359, 2008. Disponível em: <http://www.scielo.br/ scielo.php ? script=sci_arttext \& pid=S 1413 70542008000200001>. Acesso em: 18 jul. 2012. doi: 10.1590/ S1413-70542008000200001.

SILVA, W.M. et al. Emissão de $\mathrm{CO}_{2}$ e potencial de conservação de $\mathrm{C}$ em solo submetido à aplicação de diferentes adubos orgânicos. Universidade do Estado de Mato Grosso, Dourados/MT. Revista Agrarian, v.3, n.7, p.34-43, 2010.

SPARLING, G.P.; WEST, A.W. A direct extraction method to estimate soil microbial $-\mathrm{C}$ - calibration in situ using microbial respiration an ${ }^{14} \mathrm{C}$-labeled cells. Soil Biology \& Biochemistry, v.20, n.3, p.337-343, 1988. Disponível em: <http:// www.sciencedirect.com/science/article/pii/003807178 8900144>. Acesso em: 15 jul. 2012. doi: 10.1016/00380717(88)90014-4.

STOTZKY, G. Microbial respiration. In: BLACK, C.A. (Ed). Methods of soil analysis. Madison: American Society of Agronomy, 1965. p.1551-1572.

TEDESCO, M.J. et al. Análises de solo, plantas e outros materiais. 2.ed. Porto Alegre: UFRGS, Departamento de Solos, 1995. 174p.

TIRONI, S.P. et al. Efeito de herbicidas na atividade microbiana do solo. Revista Planta Daninha, v.27, p.995-1004, 2009. Disponível em: <http://www.scielo.br/scielo.php?script=sci_arttext\&pid=S010083582009000500013>. Acesso em: 15 jul. 2012. doi: 10.1590/ S0100-83582009000500013.

TÓTOLA, M.R.; CHAER, G.M. Microrganismos e processos biológicos como indicadores da qualidade dos solos. Tópicos em Ciência do Solo, v.2, p.199-275, 2002.

VANCE, E.D. et al. An extraction method for measuring soil microbial biomass C. Soil Biology \& Biochemistry, v.19, n.6, p.703-707, 1987. Disponível em: <http://www.sciencedirect.com/ science/article/pii/0038071787900526〉. Acesso em: 18 jul. 2012.

SILVA, X.F.A. et al. Biomassa microbiana e matéria orgânica leve em solos sob sistemas agrícolas orgânico e convencional na Chapada da Ibiapaba - CE. Revista Brasileira de Ciência do Solo, v.30, n.2, p.247-258, 2006. Disponível em: <http:/ /www.scielo.br/pdf/rbcs/v30n2/a06v30n2.pdf >. Acesso em: 18 jul. 2012. doi: 10.1590/S0100-06832006000200006. 